\title{
Analysis of Electric Propulsion Performance on Submersible with Motor DC, Supply Power 10260AH at Voltage 115VDC
}

\author{
Indra Ranu Kusuma ${ }^{1}$, Sardono Sarwito ${ }^{2}$, Ristita Angarini Widya Ayu Irawati ${ }^{3}$
}

\begin{abstract}
The application of diesel engine as propulsion system have some problems and weaknesses such as diesel engine unability to operate when submersible vessel is operating under sea. To overcome that problems in submersible vessel, alternative solution of ship propulsion is required. DC Motor can be used as this alternative solution. Submersible vessel use electric propulsion system with DC Motor because DC Motor has advantages of easy rotation setting and does not cause noise when submersible vessel is diving. This bachelor thesis will study the application of DC Motor as an electric propulsion system on submersible vessel with length $\mathbf{5 9 , 5 7} \mathrm{m}$ in series and parallel circuit by simulation using MATLAB software. The simulation data obtained are rotation and torque of DC Motor. From these simulation, it can be concluded that parallel circuit rotation is greater than series circuit rotation. It caused the greater speed and lower power in parallel circuit.
\end{abstract}

Keywords-DC motor, electric propulsion, MATLAB, submersible

\section{INTRODUCTION}

$\mathrm{E}$ lectric propulsion is the ship system using propulsion motor to replace performance of main engine. In general, special ship uses DC motor for propulsion propeller and merchant ship generally uses $\mathrm{AC}$ motor. DC electric motor can be used as a main propulsion and used on ships with high maneuverability, special ships, ships with a large cargo capacity, and ships using nonreversible prime movers. In general, ships use diesel engine as propulsion for propeller. But using diesel engine as propulsion for propeller have some problems and weaknesses such as diesel engine inability to operate when submersible vessel is operating under sea. To overcome that problems in submersible vessel, alternative solution of ship propulsion is required. DC Motor can be used as this alternative solution. Submersible vessel use electric propulsion system with DC Motor.

Electric propulsion system used in ship have four advantages. They are simplification of the propulsion system, silencing of onboard noise, improved fuel efficiency during travel due to the ability to constantly maintain a rotational speed that offers good fuel efficiency in the motors used for producing propulsive electric power and in the case of ships that consume extremely large amounts of power, a reduction in the financial burden of maintenance and repair including life cycle costs, due to the integration of propulsion motors with power generation motors for use in supporting general internal electrical load. Disadvantages include a higher initial cost when compared to propulsion systems

Indra Ranu Kusuma, Department of Marine Engineering, Institut Teknologi Sepuluh Nopember, Surabaya 60111, Indonesia, Email : kusuma@its.ac.id

Sardono Sarwito, Department of Marine Engineering, Institut Teknologi Sepuluh Nopember, Surabaya 60111, Indonesia, Email : sar_san@its.ac.id

Ristita Anggarini Widya Ayu Irawati, Department of Marine Engineering, Institut Teknologi Sepuluh Nopember, Surabaya 60111, Indonesia, Email : ristitaanggariniwai@gmail.com based on internal combustion engines, increased energy conversion loss from fuel to propulsion, and a larger system volume due to the large number of component parts. Electric propulsion systems are often used on ship such as icebreakers or oceanographic research vessels that take advantage of the aforementioned operational benefits, or large passenger cruise ships and others that emphasize cost and silent operation [1].

\section{METHOD}

The first step is calculating resistance on submersible vessel. The submersible has length of $59.57 \mathrm{~m}$. To calculate the resistance submersible use the formula [2]:

$$
\begin{aligned}
& \mathrm{R}_{\mathrm{BH}}=0.5 \rho \mathrm{AV}^{2} \mathrm{C}_{\mathrm{t}} \\
& \mathrm{R}_{\mathrm{APP}}=0.5 \rho \mathrm{AV}^{2} \mathrm{C}_{\mathrm{t}} \\
& \mathrm{R}_{\mathrm{T}}=\mathrm{R}_{\mathrm{BH}}+\mathrm{R}_{\mathrm{APP}}
\end{aligned}
$$

Where, $R_{t}$ is total resistance, $R_{b h}$ is bare hull resistance, $R_{\text {app }}$ is resistance of the appendages, $A$ is reference area of the submersible, $\mathrm{V}$ is velocity of the submersible, and $\mathrm{C}_{\mathrm{t}}$ is non dimensional drag coefficient. So, total resistance on the submersible is $128.594 \mathrm{kN}$.

After total resistance known, then total required power can be calculated. To calculate the torque needed on submersible use the formula:

$$
\begin{aligned}
& \mathrm{EHP}=\mathrm{Rt} \times \mathrm{V} \\
& \mathrm{THP}=((1-\mathrm{w}) /(1-\mathrm{t})) \times \mathrm{EHP} \\
& \mathrm{Q}=\mathrm{PHP} / 2 \pi \mathrm{n}
\end{aligned}
$$

Where, EHP (effective horse power) is the product total resistance and the submersible speed, Rt is total resistance, $\mathrm{V}$ is speed of the submersible, THP (thrust horse power) is the product of the thrust delivered by power from the propeller and the submersible speed, $t$ is thrust deduction, $w$ is wake fraction, $\mathrm{Q}$ is torque, PHP (propulsive horse power) is the power delivered to the ppropulsor and $\mathrm{n}$ is rotation. So, the torque required is $129 \mathrm{Nm}$. This torque will be used as a load for the DC motor when the simulation process is carried out.

Figure 1 and 2 is a circuit in a DC motor that will be simulated. Pictures of the circuit is used to create a model reference circuit in MATLAB SIMULINK software then the circuit will be run. 


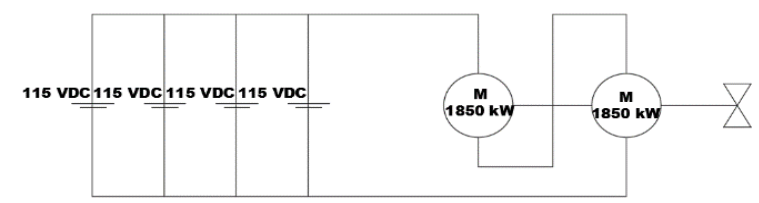

Figure. 1. DC motor series circuit to be simulated

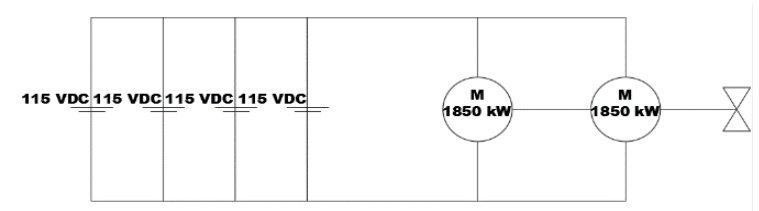

Figure. 2. DC motor parallel circuit to be simulated

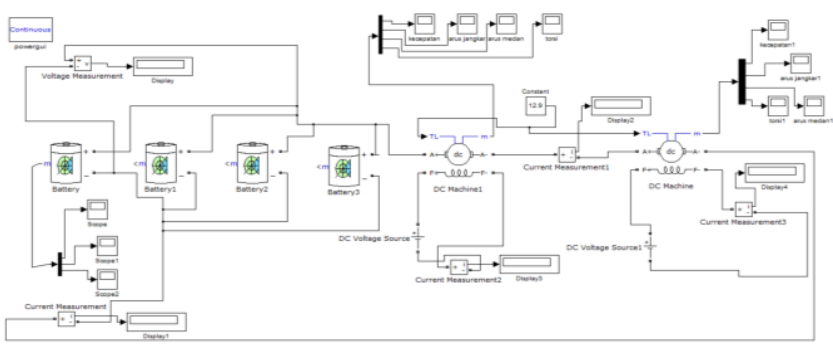

Figure. 3. DC motor series circuit in Simulink

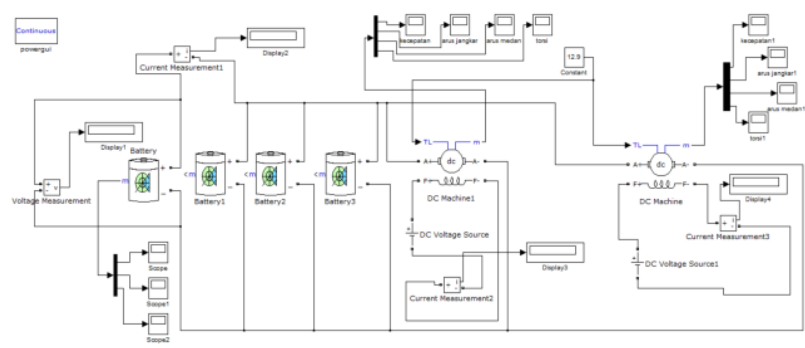

Figure. 4. DC motor parallel circuit in Simulink

Figures 3 and 4 are a series and parallel circuit of DC motor using SIMULINK. The process simulation performed for 10 seconds. Figures 5 and 6 are series and parallel of DC motor with DC-DC converter using SIMULINK. The process simulation performed for 1 seconds. The voltage on the battery is $115 \mathrm{~V}$ with a capacity of $10260 \mathrm{AH}$. Then the input torque is the torque required by the ship. Torque required the ship is $129 \mathrm{Nm}$. Series and parallel circuit in MATLAB software will be run to get the data rotation and torque on the motor with torque variation charged.

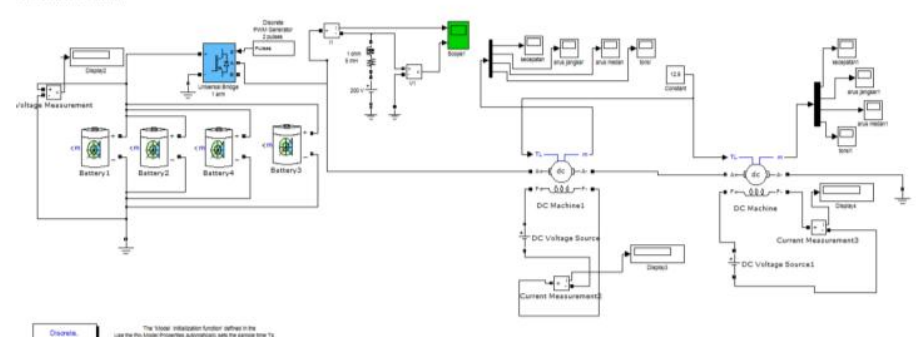

Figure. 5. DC motor series circuit with DC-DC converter in Simulink

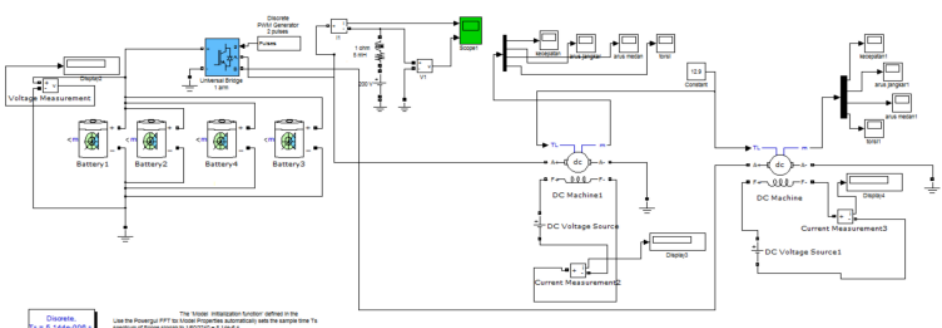

Figure. 6. DC motor parallel circuit with DC-DC converter in Simulink 
International Journal of Marine Engineering Innovation and Research, Vol. 1(2), Mar. 2017. 76-87

\section{RESULT AND DISCUSSION}

The following data obtained from the simulation results:

TABLE 1.

DATA ROTATION AND TORQUE ON SERIES CIRCUIT OF SIMULATION

\begin{tabular}{|c|c|c|c|}
\hline $\begin{array}{c}\text { Rotation } \\
(\mathrm{rad} / \mathrm{s})\end{array}$ & $\begin{array}{c}\text { Rotation } \\
(\mathrm{RPM})\end{array}$ & $\begin{array}{c}\text { Torque of } \\
\text { ship }\end{array}$ & $\begin{array}{c}\text { Torque of } \\
\text { motor }\end{array}$ \\
\hline 2,6448 & 25,25598 & 129 & 39,6 \\
\hline 2,6448 & 25,25598 & 124 & 39,1 \\
\hline 2,6449 & 25,25693 & 119 & 38,3 \\
\hline 2,6449 & 25,25693 & 114 & 37,8 \\
\hline 2,6450 & 25,25789 & 109 & 37,5 \\
\hline 2,6450 & 25,25789 & 104 & 37,1 \\
\hline 2,6451 & 25,25884 & 99 & 36,6 \\
\hline 2,6451 & 25,25884 & 94 & 36 \\
\hline 2,6452 & 25,25980 & 89 & 35,4 \\
\hline 2,6452 & 25,25980 & 84 & 34,9 \\
\hline 2,6453 & 25,26075 & 79 & 34,5 \\
\hline 2,6454 & 25,26171 & 74 & 34 \\
\hline 2,6454 & 25,26171 & 69 & 33,4 \\
\hline 2,6455 & 25,26266 & 64 & 32,8 \\
\hline 2,6455 & 25,26266 & 59 & 32,3 \\
\hline 2,6456 & 25,26362 & 54 & 32,1 \\
\hline 2,6456 & 25,26362 & 49 & 31,4 \\
\hline 2,6457 & 25,26457 & 44 & 31 \\
\hline 2,6457 & 25,26457 & 39 & 30,4 \\
\hline 2,6458 & 25,26553 & 34 & 30 \\
\hline 2,6458 & 25,26553 & 29 & 29,5 \\
\hline 2,6459 & 25,26648 & 24 & 29 \\
\hline 2,6459 & 25,26648 & 19 & 28,5 \\
\hline 2,6460 & 25,26744 & 14 & 27,9 \\
\hline 2,6460 & 25,26744 & 9 & 27,4 \\
\hline 2,6461 & 25,26839 & 4 & 27 \\
\hline 2,6461 & 25,26839 & 0 & 26,6 \\
\hline
\end{tabular}

TABLE 2 .

DATA ROTATION AND TORQUE ON PARALLEL CIRCUIT OF SIMULATION

\begin{tabular}{cccc}
$\begin{array}{c}\text { Rotation } \\
(\mathrm{rad} / \mathrm{s})\end{array}$ & $\begin{array}{c}\text { Rotation } \\
(\mathrm{RPM})\end{array}$ & $\begin{array}{c}\text { Torque of } \\
\text { ship }\end{array}$ & $\begin{array}{c}\text { Torque of } \\
\text { motor }\end{array}$ \\
\hline 5,2937 & 50,55111 & 129 & 39,4 \\
5,2937 & 50,55111 & 124 & 38,9 \\
5,2938 & 50,55207 & 119 & 38,5 \\
5,2938 & 50,55207 & 114 & 37,9 \\
5,2939 & 50,55302 & 109 & 37,4 \\
5,2939 & 50,55302 & 104 & 36,9 \\
5,2940 & 50,55398 & 99 & 36,4 \\
5,2940 & 50,55398 & 94 & 35,9 \\
5,2941 & 50,55493 & 89 & 35,4 \\
5,2941 & 50,55493 & 84 & 35,2 \\
5,2942 & 50,55589 & 79 & 34,3 \\
5,2942 & 50,55589 & 74 & 34,1 \\
5,2943 & 50,55684 & 69 & 33,6 \\
5,2943 & 50,55684 & 64 & 33,2 \\
5,2944 & 50,55780 & 59 & 32,4 \\
5,2944 & 50,55780 & 54 & 32,1 \\
5,2945 & 50,55875 & 49 & 31,4 \\
5,2946 & 50,55971 & 44 & 30,9 \\
5,2946 & 50,55971 & 39 & 30,7 \\
5,2947 & 50,56066 & 34 & 30,2 \\
5,2947 & 50,56066 & 29 & 29,6 \\
5,2948 & 50,56162 & 24 & 28,8 \\
5,2948 & 50,56162 & 19 & 28,4 \\
5,2949 & 50,56257 & 14 & 28,2 \\
5,2949 & 50,56257 & 9 & 27,6 \\
5,2950 & 50,56353 & 4 & 26,9
\end{tabular}


International Journal of Marine Engineering Innovation and Research, Vol. 1(2), Mar. 2017. 76-87 (pISSN: 2541-5972, eISSN: 2548-1479)

\begin{tabular}{llll}
5,2950 & 50,56353 & 0 & 26,7 \\
\hline
\end{tabular}

TABLE 3.

DATA ROTATION AND TORQUE ON SERIES CIRCUIT WITH DC-DC CONVERTER OF SIMULATION

\begin{tabular}{|c|c|c|c|c|}
\hline Voltage & $\begin{array}{c}\text { Rotation } \\
(\mathrm{rad} / \mathrm{s})\end{array}$ & $\begin{array}{c}\text { Rotation } \\
\text { (RPM) }\end{array}$ & $\begin{array}{l}\text { Torque } \\
\text { of ship }\end{array}$ & $\begin{array}{c}\text { Torque of } \\
\text { motor }\end{array}$ \\
\hline 134,4 & 2,4062 & 22,97752 & 10 & 64,96 \\
\hline 134,4 & 2,4061 & 22,97656 & 20 & 65,85 \\
\hline 134,4 & 2,4060 & 22,97561 & 30 & 66,96 \\
\hline 134,4 & 2,4059 & 22,97465 & 40 & 67,83 \\
\hline 134,4 & 2,4058 & 22,97370 & 50 & 68,96 \\
\hline 134,4 & 2,4057 & 22,97274 & 60 & 69,85 \\
\hline 134,4 & 2,4056 & 22,97179 & 70 & 70,96 \\
\hline 134,4 & 2,4055 & 22,97083 & 80 & 71,82 \\
\hline 134,4 & 2,4054 & 22,96988 & 90 & 72,96 \\
\hline 134,4 & 2,4053 & 22,96892 & 100 & 73,85 \\
\hline 134,4 & 2,4052 & 22,96797 & 110 & 74,96 \\
\hline 134,4 & 2,4051 & 22,96701 & 120 & 75,85 \\
\hline 134,4 & 2,4050 & 22,96606 & 129 & 76,96 \\
\hline
\end{tabular}

TABLE 4.

DATA ROTATION AND TORQUE ON CIRCUIT WITH DC-DC CONVERTER OF SIMULATION

\begin{tabular}{|c|c|c|c|c|}
\hline Voltage & $\begin{array}{c}\text { Rotation } \\
(\mathrm{rad} / \mathrm{s})\end{array}$ & $\begin{array}{c}\text { Rotation } \\
\text { (RPM) }\end{array}$ & $\begin{array}{l}\text { Torque } \\
\text { of ship }\end{array}$ & $\begin{array}{c}\text { Torque of } \\
\text { motor }\end{array}$ \\
\hline 134,4 & 4,8152 & 45,98177 & 10 & 102,46 \\
\hline 134,4 & 4,8151 & 45,98082 & 20 & 103,4 \\
\hline 134,4 & 4,8150 & 45,97986 & 30 & 104,47 \\
\hline 134,4 & 4,8149 & 45,97891 & 40 & 105,47 \\
\hline 134,4 & 4,8148 & 45,97795 & 50 & 106,4 \\
\hline 134,4 & 4,8147 & 45,97700 & 60 & 107,47 \\
\hline 134,4 & 4,8146 & 45,97604 & 70 & 108,46 \\
\hline 134,4 & 4,8145 & 45,97509 & 80 & 109,4 \\
\hline 134,4 & 4,8144 & 45,97413 & 90 & 110,47 \\
\hline 134,4 & 4,8143 & 45,97318 & 100 & 111,47 \\
\hline 134,4 & 4,8142 & 45,97222 & 110 & 112,43 \\
\hline 134,4 & 4,8141 & 45,97127 & 120 & 113,47 \\
\hline 134,4 & 4,8140 & 45,97031 & 129 & 114,47 \\
\hline
\end{tabular}

To calculate speed of ship use formula: 
International Journal of Marine Engineering Innovation and Research, Vol. 1(2), Mar. 2017. 76-87 (pISSN: 2541-5972, eISSN: 2548-1479)

$\mathrm{Vs}=\mathrm{Va} /((1-\mathrm{w}))$

The results of the calculation as follows:

TABLE 5 .

THE RESULTS OF CALCULATION OF POWER AND SPEED IN SERIES CIRCUIT

\begin{tabular}{|c|c|c|c|c|c|c|c|c|c|c|}
\hline \multicolumn{2}{|c|}{ Rotation } & \multirow{2}{*}{$\mathrm{Q}(\mathrm{Nm})$} & \multirow{2}{*}{ THP } & \multirow{2}{*}{ SHP } & \multirow{2}{*}{$\begin{array}{l}\text { BHP } \\
(\mathrm{kW})\end{array}$} & \multirow{2}{*}{$\mathrm{Va}$} & \multirow{2}{*}{$\begin{array}{c}\mathrm{Vs} \\
(\mathrm{m} / \mathrm{s})\end{array}$} & \multirow{2}{*}{$\frac{\text { Vs }}{(\text { Knot })}$} & \multirow{2}{*}{$\frac{\text { Vs }}{\text { (Knot) }}$} & \multirow{2}{*}{ Shaft Torque } \\
\hline RPM & RPS & & & & & & & & & \\
\hline 25.26839 & 0.42114 & 0 & 0.00000 & 0.00000 & 0.00000 & 0.89096 & 1.39213 & 2.70608 & 3 & 0 \\
\hline 25.26839 & 0.42114 & 4 & 10.57903 & 10.36745 & 12.19700 & 0.89096 & 1.39213 & 2.70608 & 3 & 3.92 \\
\hline 25.26744 & 0.42112 & 9 & 23.80193 & 23.32589 & 27.44222 & 0.89093 & 1.39208 & 2.70598 & 3 & 8.82 \\
\hline 25.26744 & 0.42112 & 14 & 37.02522 & 36.28472 & 42.68790 & 0.89093 & 1.39208 & 2.70598 & 3 & 13.72 \\
\hline 25.26648 & 0.42111 & 19 & 50.24661 & 49.24168 & 57.93139 & 0.89090 & 1.39203 & 2.70587 & 3 & 18.62 \\
\hline 25.26648 & 0.42111 & 24 & 63.46941 & 62.20002 & 73.17649 & 0.89090 & 1.39203 & 2.70587 & 3 & 23.52 \\
\hline 25.26553 & 0.42109 & 29 & 76.68930 & 75.15552 & 88.41825 & 0.89086 & 1.39197 & 2.70577 & 3 & 28.42 \\
\hline 25.26553 & 0.42109 & 34 & 89.91160 & 88.11336 & 103.66278 & 0.89086 & 1.39197 & 2.70577 & 3 & 33.32 \\
\hline 25.26457 & 0.42108 & 39 & 103.12999 & 101.06739 & 118.90281 & 0.89083 & 1.39192 & 2.70567 & 3 & 38.22 \\
\hline 25.26457 & 0.42108 & 44 & 116.35178 & 114.02475 & 134.14676 & 0.89083 & 1.39192 & 2.70567 & 3 & 43.12 \\
\hline 25.26362 & 0.42106 & 49 & 129.56868 & 126.97731 & 149.38507 & 0.89080 & 1.39187 & 2.70557 & 3 & 48.02 \\
\hline 25.26362 & 0.42106 & 54 & 142.78998 & 139.93418 & 164.62844 & 0.89080 & 1.39187 & 2.70557 & 3 & 52.92 \\
\hline 25.26266 & 0.42104 & 59 & 156.00537 & 152.88526 & 179.86502 & 0.89076 & 1.39181 & 2.70547 & 3 & 57.82 \\
\hline 25.26266 & 0.42104 & 64 & 169.22617 & 165.84164 & 195.10782 & 0.89076 & 1.39181 & 2.70547 & 3 & 62.72 \\
\hline 25.26171 & 0.42103 & 69 & 182.44006 & 178.79126 & 210.34266 & 0.89073 & 1.39176 & 2.70536 & 3 & 67.62 \\
\hline 25.26171 & 0.42103 & 74 & 195.66036 & 191.74715 & 225.58488 & 0.89073 & 1.39176 & 2.70536 & 3 & 72.52 \\
\hline 25.26075 & 0.42101 & 79 & 208.87276 & 204.69530 & 240.81800 & 0.89069 & 1.39171 & 2.70526 & 3 & 77.42 \\
\hline 25.25980 & 0.42100 & 84 & 222.08416 & 217.64247 & 256.04997 & 0.89066 & 1.39166 & 2.70516 & 3 & 82.32 \\
\hline 25.25980 & 0.42100 & 89 & 235.30345 & 230.59738 & 271.29104 & 0.89066 & 1.39166 & 2.70516 & 3 & 87.22 \\
\hline 25.25884 & 0.42098 & 94 & 248.51335 & 243.54308 & 286.52127 & 0.89063 & 1.39160 & 2.70506 & 3 & 92.12 \\
\hline 25.25884 & 0.42098 & 99 & 261.73215 & 256.49750 & 301.76177 & 0.89063 & 1.39160 & 2.70506 & 3 & 97.02 \\
\hline 25.25789 & 0.42096 & 104 & 274.94055 & 269.44174 & 316.99028 & 0.89059 & 1.39155 & 2.70495 & 3 & 101.92 \\
\hline 25.25789 & 0.42096 & 109 & 288.15884 & 282.39567 & 332.23019 & 0.89059 & 1.39155 & 2.70495 & 3 & 106.82 \\
\hline 25.25693 & 0.42095 & 114 & 301.36574 & 295.33843 & 347.45697 & 0.89056 & 1.39150 & 2.70485 & 3 & 111.72 \\
\hline 25.25693 & 0.42095 & 119 & 314.58354 & 308.29187 & 362.69632 & 0.89056 & 1.39150 & 2.70485 & 3 & 116.62 \\
\hline 25.25598 & 0.42093 & 124 & 327.78894 & 321.23316 & 377.92137 & 0.89053 & 1.39145 & 2.70475 & 3 & 121.52 \\
\hline 25.25598 & 0.42093 & 129 & 341.00624 & 334.18611 & 393.16013 & 0.89053 & 1.39145 & 2.70475 & 3 & 0 \\
\hline
\end{tabular}


TABLE 6.

THE RESULTS OF CALCULATION OF POWER AND SPEED IN PARALLEL CIRCUIT

\begin{tabular}{|c|c|c|c|c|c|c|c|c|c|c|}
\hline \multicolumn{2}{|c|}{ Rotation } & \multirow{2}{*}{$\mathrm{Q}(\mathrm{Nm})$} & \multirow{2}{*}{ THP } & \multirow{2}{*}{ SHP } & \multirow{2}{*}{$\begin{array}{l}\text { BHP } \\
(\mathrm{kW})\end{array}$} & \multirow{2}{*}{$\mathrm{Va}$} & \multirow{2}{*}{$\frac{\mathrm{Vs}}{(\mathrm{m} / \mathrm{s})}$} & \multirow{2}{*}{$\begin{array}{c}\text { Vs } \\
\text { (Knot) }\end{array}$} & \multirow{2}{*}{$\frac{\text { Vs }}{\text { (Knot) }}$} & \multirow{2}{*}{ Shaft Torque } \\
\hline RPM & RPS & & & & & & & & & \\
\hline 50.56353 & 0.84273 & 0 & 0.00000 & 0.00000 & 0.00000 & 1.78287 & 2.78573 & 5.41502 & 5 & 0 \\
\hline 50.56353 & 0.84273 & 4 & 21.16926 & 20.74588 & 24.40691 & 1.78287 & 2.78573 & 5.41502 & 5 & 3.92 \\
\hline 50.56257 & 0.84271 & 9 & 47.62994 & 46.67734 & 54.91452 & 1.78284 & 2.78568 & 5.41492 & 5 & 8.82 \\
\hline 50.56257 & 0.84271 & 14 & 74.09102 & 72.60920 & 85.42259 & 1.78284 & 2.78568 & 5.41492 & 5 & 13.72 \\
\hline 50.56162 & 0.84269 & 19 & 100.55020 & 98.53920 & 115.92847 & 1.78280 & 2.78563 & 5.41482 & 5 & 18.62 \\
\hline 50.56162 & 0.84269 & 24 & 127.01078 & 124.47056 & 146.43596 & 1.78280 & 2.78563 & 5.41482 & 5 & 23.52 \\
\hline 50.56066 & 0.84268 & 29 & 153.46846 & 150.39909 & 176.94011 & 1.78277 & 2.78558 & 5.41471 & 5 & 28.42 \\
\hline 50.56066 & 0.84268 & 34 & 179.92854 & 176.32997 & 207.44702 & 1.78277 & 2.78558 & 5.41471 & 5 & 33.32 \\
\hline 50.55971 & 0.84266 & 39 & 206.38472 & 202.25702 & 237.94944 & 1.78274 & 2.78552 & 5.41461 & 5 & 38.22 \\
\hline 50.55971 & 0.84266 & 44 & 232.84430 & 228.18741 & 268.45578 & 1.78274 & 2.78552 & 5.41461 & 5 & 43.12 \\
\hline 50.55875 & 0.84265 & 49 & 259.29898 & 254.11300 & 298.95647 & 1.78270 & 2.78547 & 5.41451 & 5 & 48.02 \\
\hline 50.55780 & 0.84263 & 54 & 285.75266 & 280.03761 & 329.45601 & 1.78267 & 2.78542 & 5.41441 & 5 & 52.92 \\
\hline 50.55780 & 0.84263 & 59 & 312.21124 & 305.96702 & 359.96120 & 1.78267 & 2.78542 & 5.41441 & 5 & 57.82 \\
\hline 50.55684 & 0.84261 & 64 & 338.66343 & 331.89016 & 390.45901 & 1.78263 & 2.78537 & 5.41431 & 5 & 62.72 \\
\hline 50.55684 & 0.84261 & 69 & 365.12151 & 357.81908 & 420.96362 & 1.78263 & 2.78537 & 5.41431 & 5 & 67.62 \\
\hline 50.55589 & 0.84260 & 74 & 391.57219 & 383.74075 & 451.45970 & 1.78260 & 2.78531 & 5.41420 & 5 & 72.52 \\
\hline 50.55589 & 0.84260 & 79 & 418.02977 & 409.66917 & 481.96373 & 1.78260 & 2.78531 & 5.41420 & 5 & 77.42 \\
\hline 50.55493 & 0.84258 & 84 & 444.47895 & 435.58938 & 512.45809 & 1.78257 & 2.78526 & 5.41410 & 5 & 82.32 \\
\hline 50.55493 & 0.84258 & 89 & 470.93603 & 461.51731 & 542.96155 & 1.78257 & 2.78526 & 5.41410 & 5 & 87.22 \\
\hline 50.55398 & 0.84257 & 94 & 497.38372 & 487.43605 & 573.45417 & 1.78253 & 2.78521 & 5.41400 & 5 & 92.12 \\
\hline 50.55398 & 0.84257 & 99 & 523.84030 & 513.36350 & 603.95705 & 1.78253 & 2.78521 & 5.41400 & 5 & 97.02 \\
\hline 50.55302 & 0.84255 & 104 & 550.28649 & 539.28076 & 634.44795 & 1.78250 & 2.78516 & 5.41390 & 5 & 101.92 \\
\hline 50.55302 & 0.84255 & 109 & 576.74257 & 565.20772 & 664.95026 & 1.78250 & 2.78516 & 5.41390 & 5 & 106.82 \\
\hline 50.55207 & 0.84253 & 114 & 603.18726 & 591.12351 & 695.43942 & 1.78247 & 2.78510 & 5.41379 & 5 & 111.72 \\
\hline 50.55207 & 0.84253 & 119 & 629.64284 & 617.04998 & 725.94115 & 1.78247 & 2.78510 & 5.41379 & 5 & 116.62 \\
\hline 50.55111 & 0.84252 & 124 & 656.08602 & 642.96430 & 756.42859 & 1.78243 & 2.78505 & 5.41369 & 5 & 121.52 \\
\hline 50.55111 & 0.84252 & 129 & 682.54111 & 668.89028 & 786.92975 & 1.78243 & 2.78505 & 5.41369 & 5 & 126.42 \\
\hline
\end{tabular}

TABLE 7.

THE RESULTS OF CALCULATION OF POWER AND SPEED IN SERIES CIRCUIT WITH DC-DC CONVERTER

\begin{tabular}{|c|c|c|c|c|c|c|c|c|c|c|}
\hline \multicolumn{2}{|c|}{ Rotation } & \multirow{2}{*}{$\mathrm{Q}(\mathrm{Nm})$} & \multirow{2}{*}{ THP } & \multirow{2}{*}{ SHP } & \multirow{2}{*}{$\frac{\mathrm{BHP}}{(\mathrm{kW})}$} & \multirow{2}{*}{$\mathrm{Va}$} & \multirow{2}{*}{$\frac{\mathrm{Vs}}{(\mathrm{m} / \mathrm{s})}$} & \multirow{2}{*}{$\frac{\text { Vs }}{\text { (Knot) }}$} & \multirow{2}{*}{$\frac{\mathrm{Vs}}{(\mathrm{Knot})}$} & \multirow{2}{*}{ Shaft Torque } \\
\hline RPM & RPS & & & & & & & & & \\
\hline 22.97752 & 0.38296 & 10 & 24.04980 & 23.56881 & 27.72801 & 0.81019 & 1.26592 & 2.46074 & 2 & 9.8 \\
\hline 22.97656 & 0.38294 & 20 & 48.09760 & 47.13565 & 55.45371 & 0.81015 & 1.26586 & 2.46064 & 2 & 19.6 \\
\hline 22.97561 & 0.38293 & 30 & 72.14341 & 70.70054 & 83.17711 & 0.81012 & 1.26581 & 2.46054 & 2 & 29.4 \\
\hline 22.97465 & 0.38291 & 40 & 96.18721 & 94.26347 & 110.89820 & 0.81009 & 1.26576 & 2.46043 & 2 & 39.2 \\
\hline 22.97370 & 0.38289 & 50 & 120.22902 & 117.82444 & 138.61699 & 0.81005 & 1.26571 & 2.46033 & 2 & 49 \\
\hline 22.97274 & 0.38288 & 60 & 144.26882 & 141.38345 & 166.33347 & 0.81002 & 1.26565 & 2.46023 & 2 & 58.8 \\
\hline 22.97179 & 0.38286 & 70 & 168.30663 & 164.94050 & 194.04765 & 0.80999 & 1.26560 & 2.46013 & 2 & 68.6 \\
\hline 22.97083 & 0.38285 & 80 & 192.34244 & 188.49559 & 221.75952 & 0.80995 & 1.26555 & 2.46003 & 2 & 78.4 \\
\hline 22.96988 & 0.38283 & 90 & 216.37625 & 212.04873 & 249.46909 & 0.80992 & 1.26550 & 2.45992 & 2 & 88.2 \\
\hline 22.96892 & 0.38282 & 100 & 240.40806 & 235.59990 & 277.17635 & 0.80988 & 1.26544 & 2.45982 & 2 & 98 \\
\hline 22.96797 & 0.38280 & 110 & 264.43787 & 259.14912 & 304.88131 & 0.80985 & 1.26539 & 2.45972 & 2 & 107.8 \\
\hline 22.96701 & 0.38278 & 120 & 288.46569 & 282.69637 & 332.58397 & 0.80982 & 1.26534 & 2.45962 & 2 & 117.6 \\
\hline 22.96606 & 0.38277 & 129 & 310.08772 & 303.88596 & 357.51290 & 0.80978 & 1.26529 & 2.45951 & 2 & 126.42 \\
\hline
\end{tabular}


International Journal of Marine Engineering Innovation and Research, Vol. 1(2), Mar. 2017. 76-87 (pISSN: 2541-5972, eISSN: 2548-1479)

TABLE 8 .

THE RESULTS OF CALCULATION OF POWER AND SPEED IN PARALLEL CIRCUIT WITH DC-DC CONVERTER

\begin{tabular}{|c|c|c|c|c|c|c|c|c|c|c|}
\hline \multicolumn{2}{|c|}{ Rotation } & \multirow{2}{*}{$\mathrm{Q}(\mathrm{Nm})$} & \multirow{2}{*}{ THP } & \multirow{2}{*}{ SHP } & \multirow{2}{*}{$\frac{\mathrm{BHP}}{(\mathrm{kW})}$} & \multirow{2}{*}{$\mathrm{Va}$} & \multirow{2}{*}{$\frac{\mathrm{Vs}}{(\mathrm{m} / \mathrm{s})}$} & \multirow{2}{*}{$\frac{\text { Vs }}{\text { (Knot) }}$} & \multirow{2}{*}{$\frac{\mathrm{Vs}}{(\mathrm{Knot})}$} & \multirow{2}{*}{ Shaft Torque } \\
\hline RPM & RPS & & & & & & & & & \\
\hline 45.98177 & 0.76636 & 10 & 48.12759 & 47.16504 & 55.48828 & 1.62132 & 2.53331 & 4.92435 & 5 & 9.8 \\
\hline 45.98082 & 0.76635 & 20 & 96.25318 & 94.32812 & 110.97425 & 1.62128 & 2.53326 & 4.92424 & 5 & 19.6 \\
\hline 45.97986 & 0.76633 & 30 & 144.37677 & 141.48923 & 166.45792 & 1.62125 & 2.53320 & 4.92414 & 5 & 29.4 \\
\hline 45.97891 & 0.76632 & 40 & 192.49836 & 188.64840 & 221.93929 & 1.62122 & 2.53315 & 4.92404 & 5 & 39.2 \\
\hline 45.97795 & 0.76630 & 50 & 240.61796 & 235.80560 & 277.41835 & 1.62118 & 2.53310 & 4.92394 & 5 & 49 \\
\hline 45.97700 & 0.76628 & 60 & 288.73555 & 282.96084 & 332.89510 & 1.62115 & 2.53305 & 4.92383 & 5 & 58.8 \\
\hline 45.97604 & 0.76627 & 70 & 336.85114 & 330.11412 & 388.36955 & 1.62112 & 2.53299 & 4.92373 & 5 & 68.6 \\
\hline 45.97509 & 0.76625 & 80 & 384.96474 & 377.26545 & 443.84170 & 1.62108 & 2.53294 & 4.92363 & 5 & 78.4 \\
\hline 45.97413 & 0.76624 & 90 & 433.07634 & 424.41481 & 499.31154 & 1.62105 & 2.53289 & 4.92353 & 5 & 88.2 \\
\hline 45.97318 & 0.76622 & 100 & 481.18594 & 471.56222 & 554.77908 & 1.62101 & 2.53283 & 4.92343 & 5 & 98 \\
\hline 45.97222 & 0.76620 & 110 & 529.29354 & 518.70766 & 610.24431 & 1.62098 & 2.53278 & 4.92332 & 5 & 107.8 \\
\hline 45.97127 & 0.76619 & 120 & 577.39914 & 565.85115 & 665.70724 & 1.62095 & 2.53273 & 4.92322 & 5 & 117.6 \\
\hline 45.97031 & 0.76617 & 129 & 620.69118 & 608.27735 & 715.62042 & 1.62091 & 2.53268 & 4.92312 & 5 & 126.42 \\
\hline
\end{tabular}

Silent mode operation is an operation used by submersibles when they want to infiltrate enemy territory or to hide from the enemy. Silent operation mode has a speed of 1 knot up to 6 knots. So the series circuit has been simulated to have a speed of 2 until 3 knots, the speed is usually used to hide from enemies. Because with that speed, vibrations produced is small, therefore it is easier to be undetected by the enemy. Parallel circuit has a speed of 5 knots that are usually used to infiltrate enemy territory due to speed of 5 knots that usually can not be detected by enemy. Therefore, the opponent can get into opponent's area and opponent can use it to attack enemy territory.

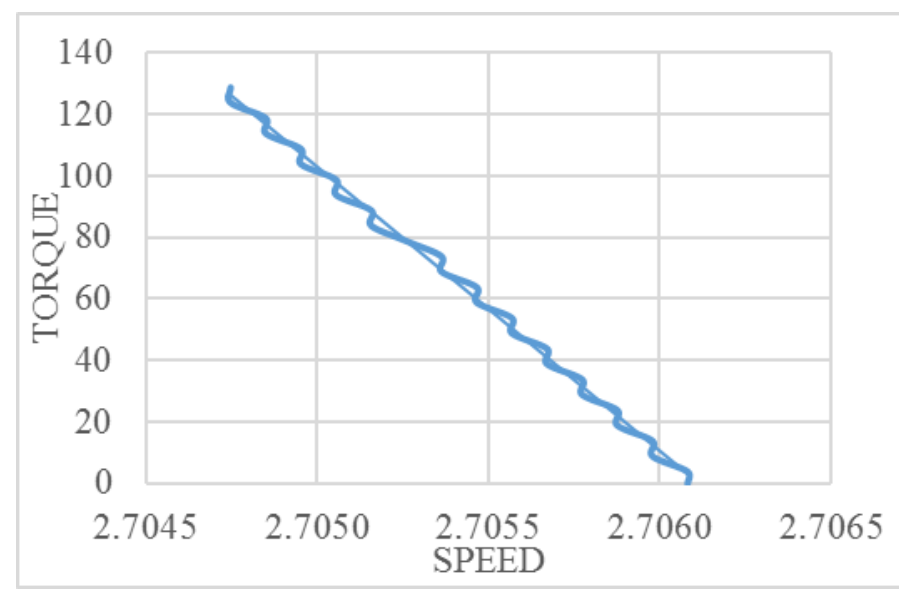

Figure. 7. Graph of speed versus torque in series circuit 


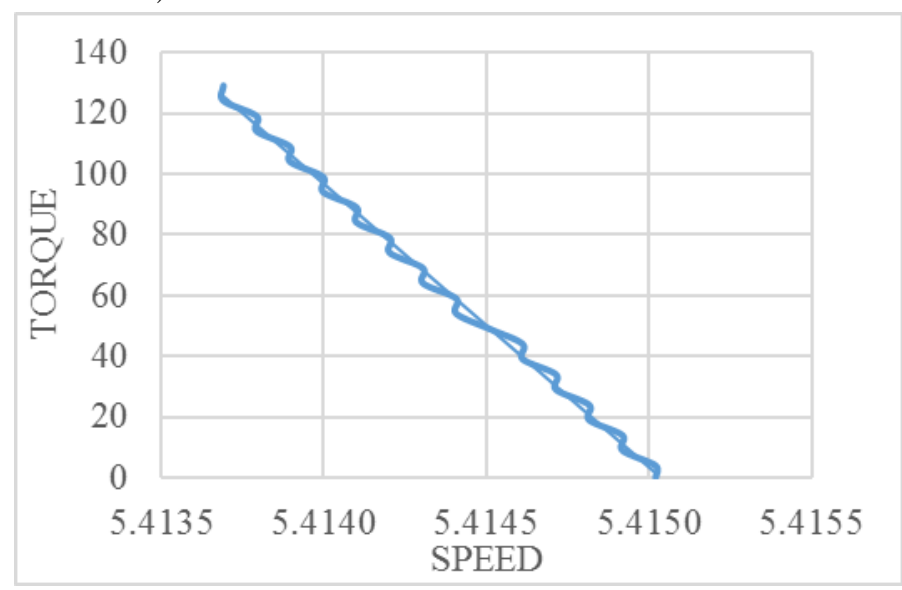

Figure. 8. Graph of speed versus torque in parallel circuit

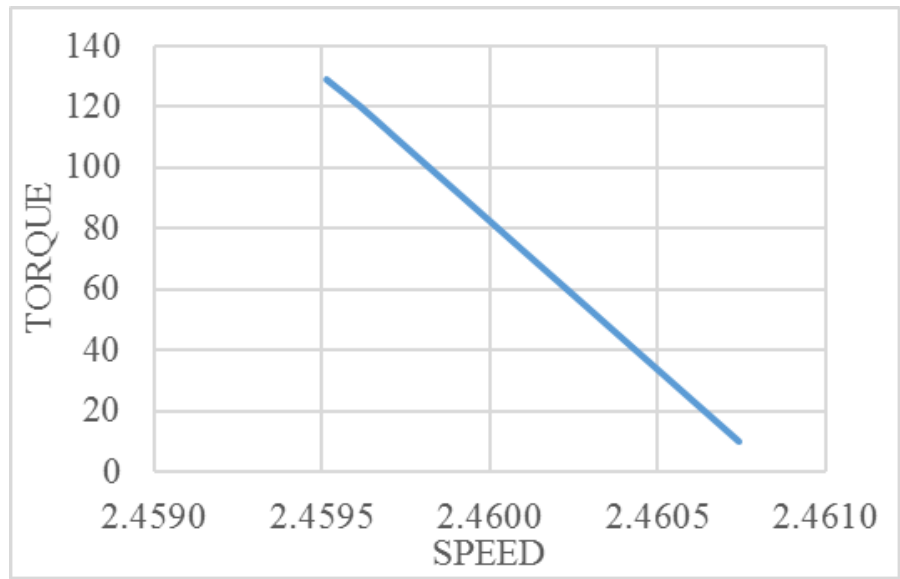

Figure. 9. Graph of speed versus torque in series circuit with DC-DC converter

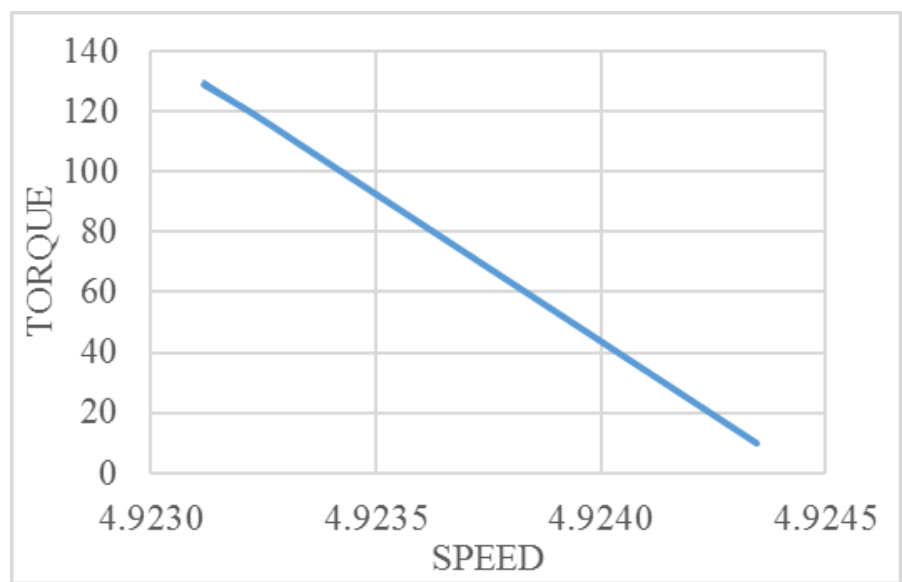

Figure. 10. Graph of speed versus torque in parallel circuit with DC-DC converter

Figure 7, 8, 9 and 10 are the graph showing the relationship between the speed of the torque in series and parallel circuits when the battery is full. Based on the graph, the torque value is inversely proportional to the value of speed. In series circuit, when the speed of the vessel reached its low point of 2.70475 knots then the value of torque produced is the highest of $129 \mathrm{Nm}$. Meanwhile, when the maximum speed is 2.70608 knots then the value of torque produced is the lowest $0 \mathrm{Nm}$. While parallel circuit, when the speed of the ship reached its low point of 5.41369 knots then the value of torque produced is the highest of $129 \mathrm{Nm}$. Meanwhile, when the maximum speed is 5.41502 knots then the value of torque produced is the lowest $0 \mathrm{Nm}$. In series circuit with DC-DC converter, when the speed of the vessel reached its low point of 2.45951 knots then the value of torque produced is the highest of $129 \mathrm{Nm}$. Meanwhile, when the maximum speed is 2.46074 knots then the value of torque produced is the lowest $10 \mathrm{Nm}$. While parallel circuit with DC-DC conveter, when the speed of the ship reached its low point of 4.92312 knots then the value of torque produced is the highest of $129 \mathrm{Nm}$. Meanwhile, when the maximum speed is 4.92435 knots then the value of torque produced is the lowest $10 \mathrm{Nm}$. Thus, the torque is directly proportional to Ia. 
International Journal of Marine Engineering Innovation and Research, Vol. 1(2), Mar. 2017. 76-87 (pISSN: 2541-5972, eISSN: 2548-1479)

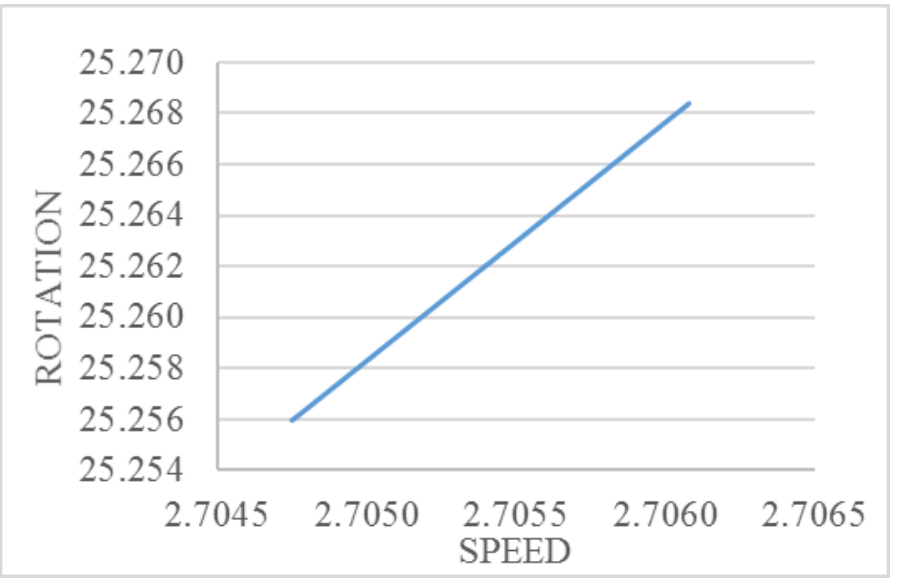

Figure. 11. Graph of speed versus rotation in series circuit

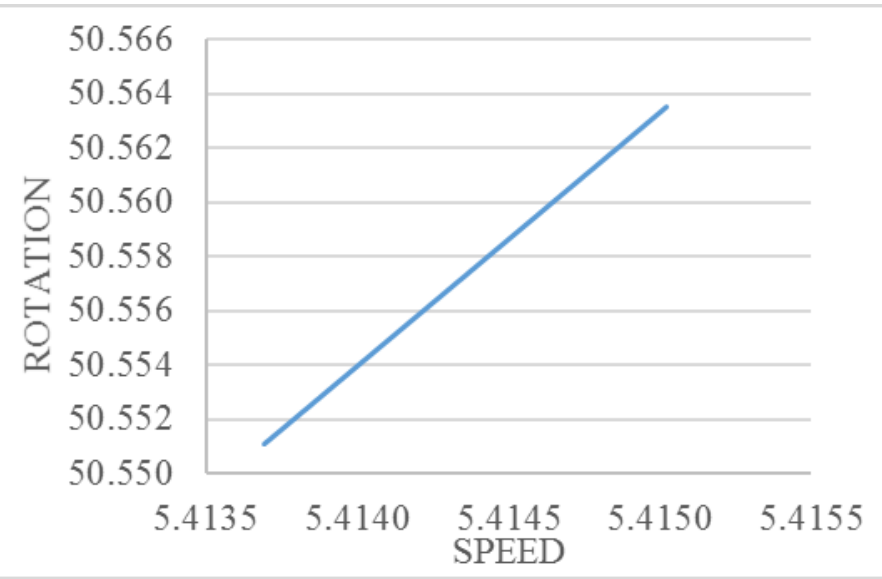

Figure. 12. Graph of speed versus rotation in parallel circuit

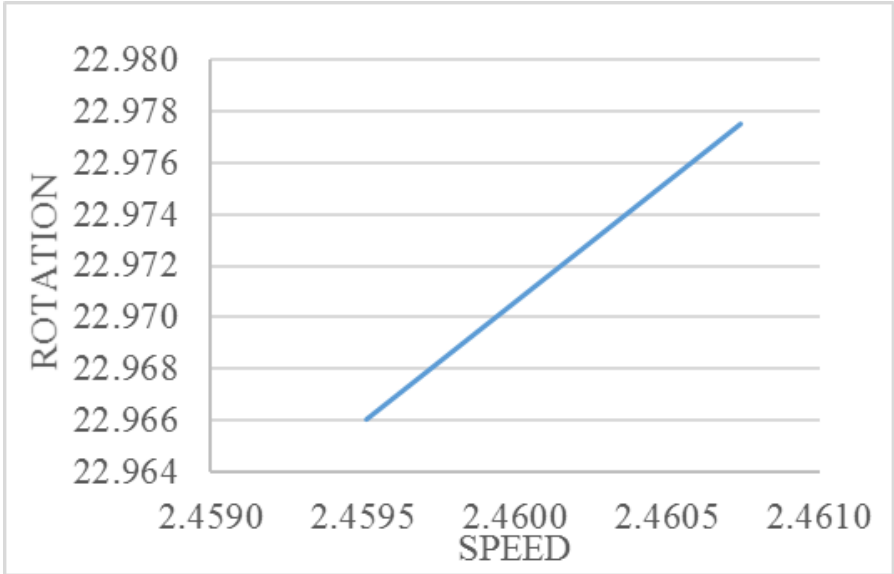

Figure. 13. Graph of speed versus rotation in series circuit with DC-DC converter

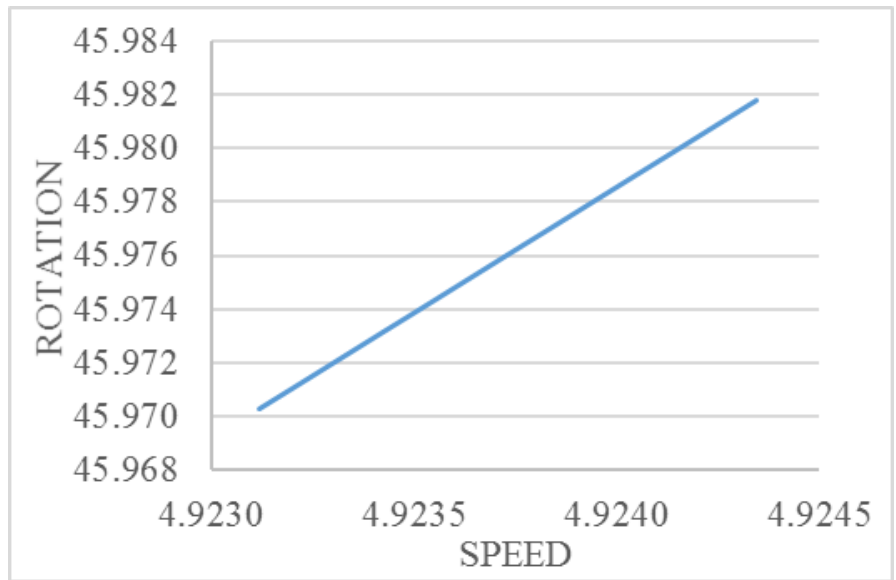

Figure. 14. Graph of speed versus rotation in parallel circuit with DC-DC converter 
Figure 11, 12, 13 and 14 are graph showing the relationship between the speed of the rotation in the series and parallel circuits when the battery is full. In a series circuit, when the speed 2.70475 knots, the rotation worth $25.25598 \mathrm{rpm}$ and when the speed 2.70608 knots, the rotation worth $25.26839 \mathrm{rpm}$. In a parallel circuit, when the speed 5.41502 knots, the rotation worth $50.56353 \mathrm{rpm}$ and when the speed 5.41369 knots, the rotation worth $50.55111 \mathrm{rpm}$. In a series circuit with DCDC converter, when the speed 2.45951 knots, the rotation worth $25.96606 \mathrm{rpm}$ and when the speed 2.46074 knots, the rotation $22.97752 \mathrm{rpm}$. In a parallel circuit with DC-DC converter, when the speed 4.92312 knots, the rotation worth $45.97031 \mathrm{rpm}$ and when the speed 4.92435 knots, the rotation worth $45.98177 \mathrm{rpm}$. When a ship's speed is maximum speed, the rotation is maximum too. Conversely, if the speed is minimum, the rotation generated is also minimum. Thus, the relationship between speed and the rotation is directly proportional.

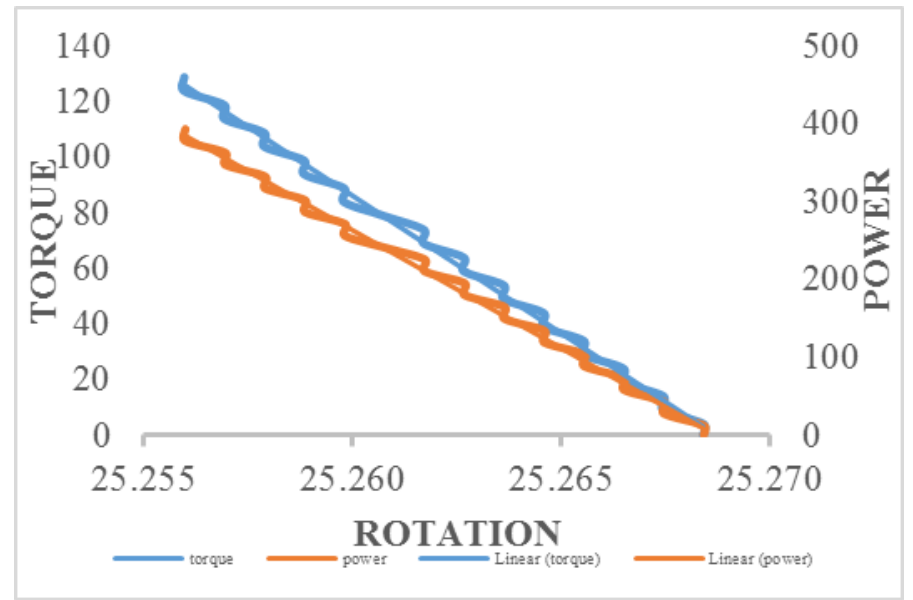

Figure. 15. Graph of rotation versus torque and power in series circuit

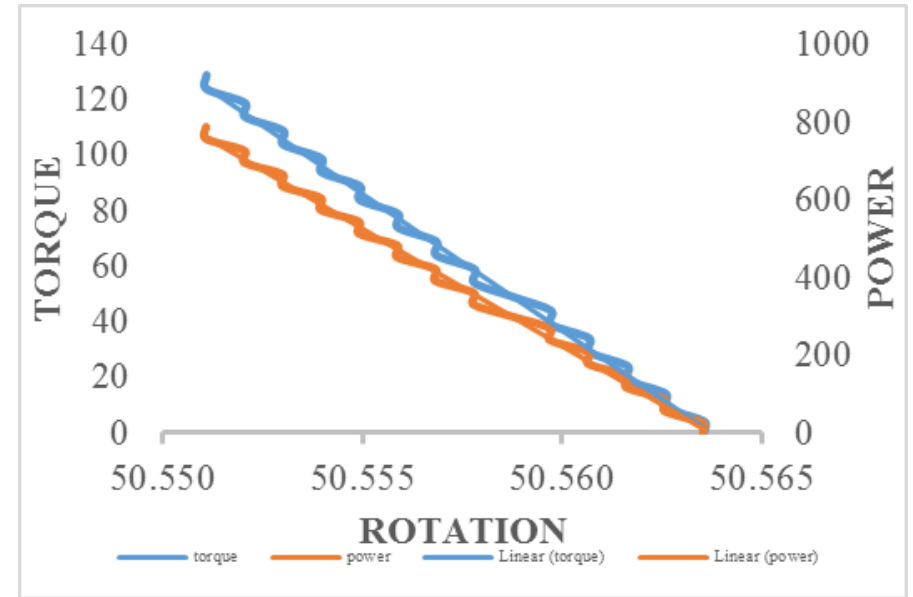

Figure. 16. Graph of rotation versus torque and power in parallel circuit

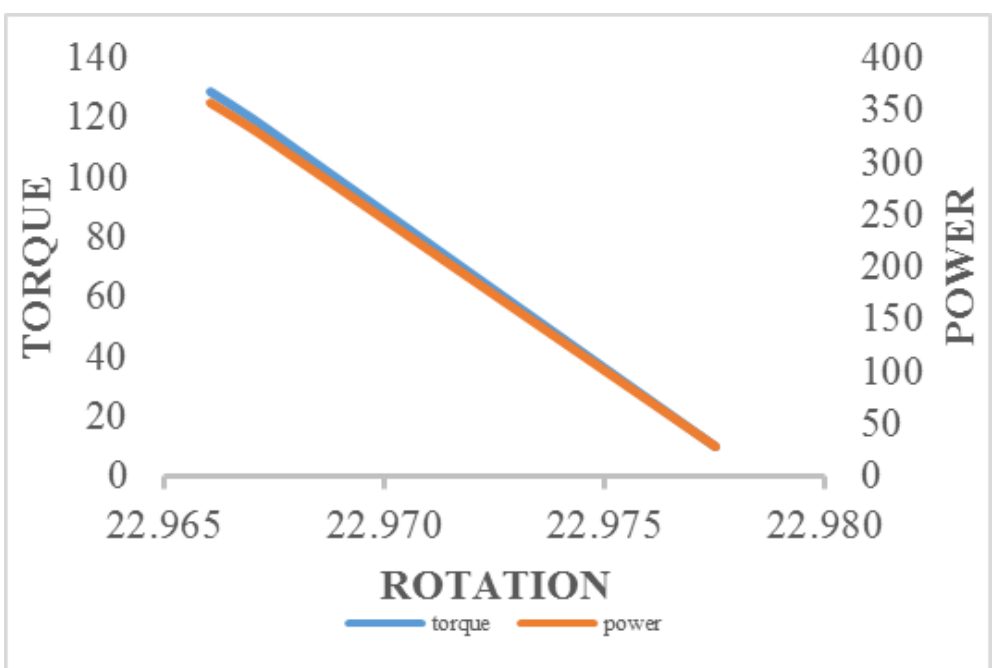

Figure. 17. Graph of rotation versus torque and power in series circuit with DC-DC converter 


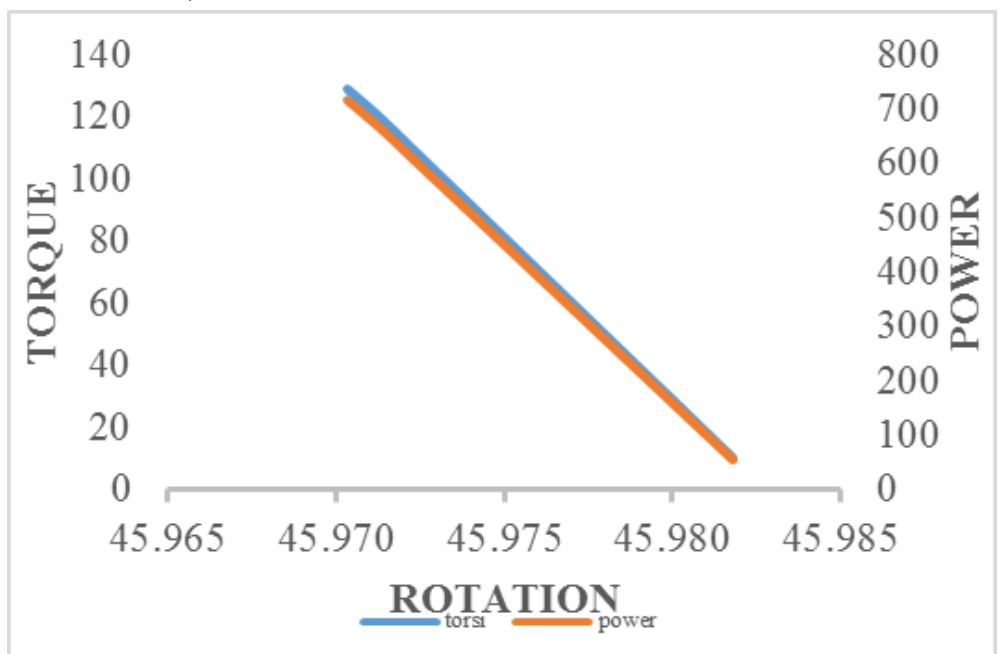

Figure. 18. Graph of rotation versus torque and power in parallel circuit with DC-DC converter

Figure 15, 16, 17 and 18 shows graph of rotation versus torque and power at the series and parallel circuits when the battery is full. In series circuit, when value of torque is $129 \mathrm{Nm}$, the rotation is $25.25598 \mathrm{rpm}$ and power is $393.16013 \mathrm{~kW}$. In parallel circuit, when value of torque is $129 \mathrm{Nm}$, the rotation is $50.55111 \mathrm{rpm}$ and power is $786.92975 \mathrm{~kW}$. In series circuit with DC-DC converter, when value of torque is $129 \mathrm{Nm}$, the rotation is $22.96606 \mathrm{rpm}$ and power is $357.51290 \mathrm{~kW}$. In parallel circuit with DC-DC converter, when value of torque is $129 \mathrm{Nm}$, the rotation is $45.97031 \mathrm{rpm}$ and power is $715.62042 \mathrm{~kW}$. So, rotation is inversely proportional to the torque and the highest value of rotation require the smallest power.

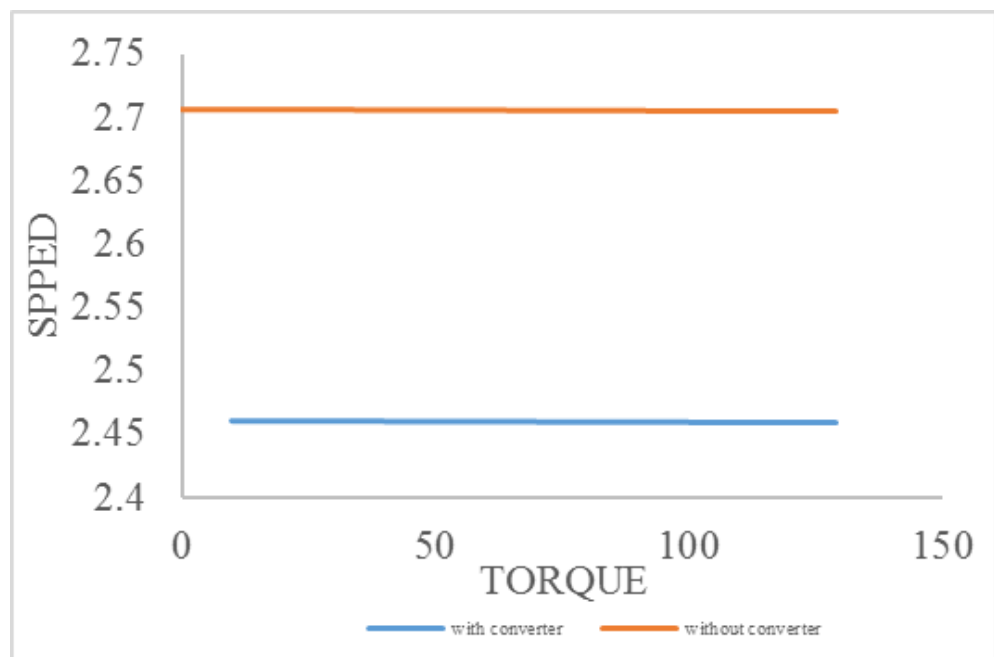

Figure. 19. Graph of torque versus speed in series circuit with and without DC-DC converter

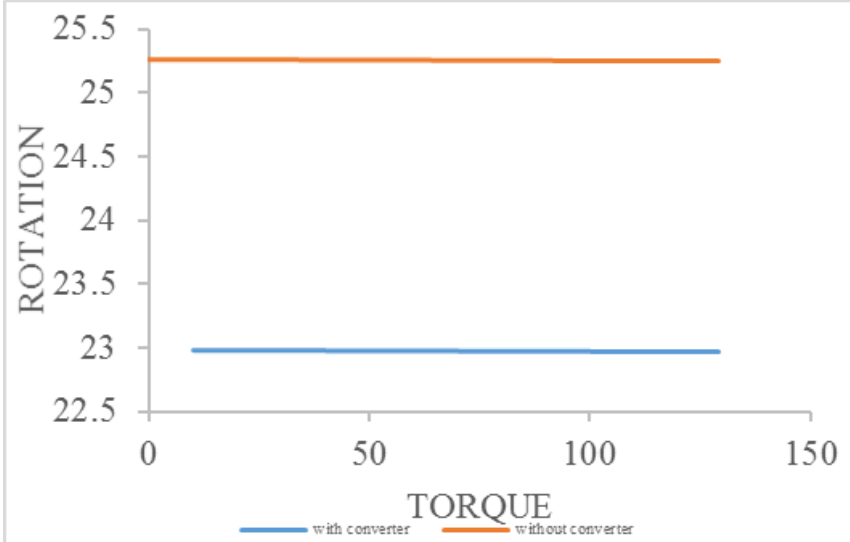

Figure. 20. Graph of torque versus speed in series circuit with and without DC-DC converter 


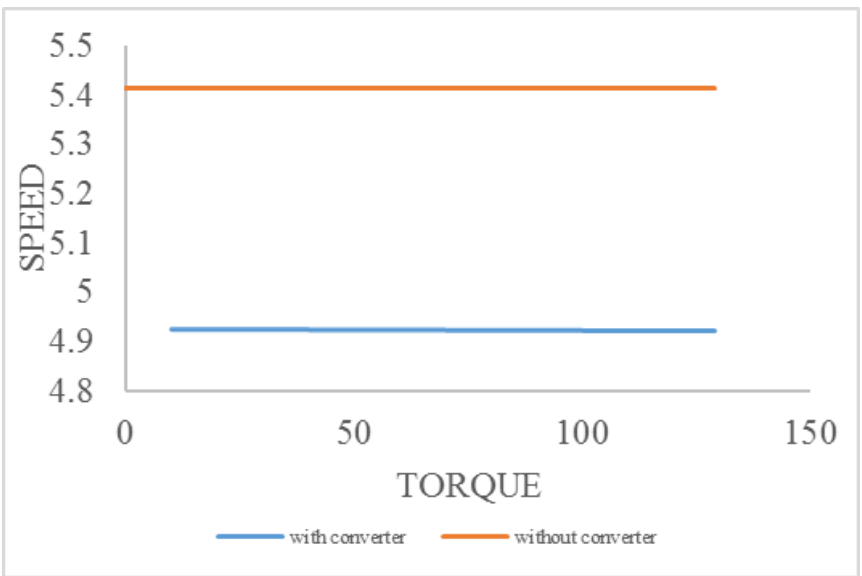

Figure. 21. Graph of torque versus speed in parallel circuit with and without DC-DC converter

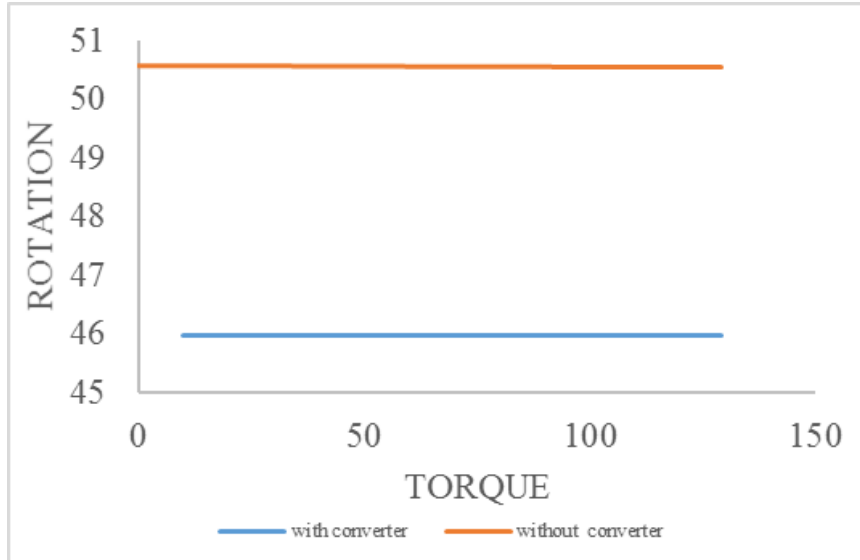

Figure. 22. Graph of torque versus speed in parallel circuit with and without DC-DC converter

Figure 19, 20, 21 and 22 show different speed and torque with and without DC-DC converter in series and parallel circuit when battery is full. Series circuit without DC-DC converter has speed 2,46 knot and rotation 23 rpm. Series circuit with DC-DC converter has speed 2.7 knot and rotation $25.26 \mathrm{rpm}$. Parallel circuit without DCDC converter has speed 5.41 knot and rotation 50.55 rpm. Parallel circuit with DC-DC converter has speed 4.92 knot and rotation $45.97 \mathrm{rpm}$. Circuits with DC-DC converter have smaller speed and rotation than circuits without DC-DC converter.

\section{CONCLUSION}

From these process simulation and analysis graphs can be concluded that if the speed increases, the torque generated will decrease. So the relationship between the torque is inversely proportional to the speed.

When a ship's speed is maximum speed, the rotation is maximum too. Conversely, if the speed is minimum, the rotation generated is also minimum. Thus, the relationship between speed and the rotation is directly proportional.

The greater value of the torque need smaller the rotation produced by the motor and more power is needed.

Circuits with DC-DC converter have smaller speed and rotation than circuits without DC-DC converter.

The series circuit simulated has a speed of 2 until 3 knots and is usually used to hide from opponent. While the parallel circuit has a speed of 5 until 5.4 knots and is usually used to infiltrate opponent's area.

\section{REFERENCES}

[1] Yoshifumi Ajioka and Kiyoshi Ohno, "Electric propulsion systems for ships", Hitachi Review Vol. 62 (2013), No. 3, pp:231-232.

[2] Eugene Allmendinger, "Submersible vehicle systems design", Publish: The Society of Naval Architects and Marine Engineers, 1990, pp.252-258. 